\title{
Nonthermal X-rays from supernova remnants
}

\author{
Anne Decourchelle \\ Laboratoire AIM, CEA-IRFU/CNRS/ Univ. Paris Diderot, Service d'Astrophysique, \\ L'Orme des Merisiers, CEA Saclay \\ F-91191 Gif sur Yvette, France \\ email: anne.decourchelle@cea.fr
}

\begin{abstract}
Since the discovery of nonthermal X-rays in the shell-type supernova remnant SN1006 almost 20 years ago, the field has developed considerably, owing significant progress to our understanding of particle acceleration. Key to the characterization of the nonthermal emission is the ability of current satellites, XMM-Newton and Chandra, to perform spatially resolved spectroscopy at a relatively small spatial scale.

In this review, I intend to present the main contributions of the study of nonthermal X-rays from supernova remnants to the understanding of particle acceleration.
\end{abstract}

Keywords. Supernova remnant, Particle acceleration, X-rays, Synchrotron emission

\section{Introduction}

Following the explosion, the supernova material (ejecta) is ejected at high velocity (a few tens of thousands of $\mathrm{km} / \mathrm{s}$ ) in the circumstellar medium and experiences a rapid expansion. As the expansion velocity is much larger than the sound speed, the ejecta are preceded by a shock, called forward shock that propagates outwards and compresses, heats and accelerates the ambient medium. After an initial free expansion phase, the ejecta are decelerated giving rise to a reverse shock, which propagates inwards and compresses, heats and decelerates the ejecta. The result of the interaction of high-velocity ejecta with the surrounding medium is thus a two-shock structure as is illustrated in Fig. 1. The interface between the ejecta and the ambient medium is distorted due to hydrodynamic instabilities (notably, Rayleigh-Taylor). The two shocked media are expected to be powerful X-ray emitters, as they have temperatures of tens of million degrees and above. It is in this early ejecta-dominated phase (for remnants younger than typically 1000 years) that the elements synthesized in the supernovae are best observable, notably in X-rays, as the thermal emission is dominated by that of the shocked ejected matter (see Slane 2013, this proceedings).

On theoretical grounds, strong shocks in supernova remnants are expected to be responsible for the acceleration of Galactic cosmic rays up to the knee at about $310^{15} \mathrm{eV}$ (Blandford \& Eichler 1987). This is the only galactic population fitting the energetic requirement to maintain the pool of Galactic cosmic rays, assuming that about $10 \%$ of their kinetic energy is taken by the acceleration process. The presence of $\mathrm{GeV}$ accelerated electrons at the shocks in supernova remnants is known since the discovery of synchrotron radio emission in Tycho in 1954 (Hanbury Brown 1954) and is now observed in almost all SNRs. The spectral index of their radio synchrotron spectrum is close to the theoretical expectation. But the search for observational proofs of electrons accelerated close to the knee has failed for a long time in the high-energy domain.

Indeed, synchrotron X-rays were observed only 40 years later by the $A S C A$ Japanese satellite in the shell-like remnant of SN 1006 (Koyama et al. 1995), thanks to the achievement of CCD X-ray spectro-imagers. It has allowed obtaining the first evidence of 
electrons accelerated up to $\mathrm{TeV}$ energies in the remnant of SN 1006. The bright X-ray and radio limbs were indeed revealed to be featureless in X-rays, and interpreted as Xray synchrotron emission from $\mathrm{TeV}$ electrons. Conversely, the emission in the faint areas (center of the remnant and faint limbs), exhibiting emission lines, are of thermal origin. This result, as well as the subsequent discovery of two other synchrotron-dominated supernova remnants, have shown the new potential of X-ray observations to observe the highest-energy electrons that shocks can accelerate in supernova remnants (SNRs).

As illustrated in Fig. 1, accelerated particles give rise to a number of emission processes through their interaction with the magnetic field (synchrotron, from radio to X-rays), with the ambient photon field (inverse Compton scattering, in the gamma-ray band) and with the ambient medium (pion decay, in the gamma-ray domain). A full multiwavelength nonthermal approach is important to consistently derive the characteristics of the accelerated particles and of their environment. This includes using combined radio and X-ray data to constrain the synchrotron spectrum, as well as very high-energy gamma rays whose inverse-Compton emission arises from the $\mathrm{X}$-ray synchrotron emitting electron population. The very high-energy gamma-ray domain (see Lemoine-Goumard 2013, this proceedings) has considerably developed in the last ten years, with the implementation of stereoscopic ground-based Cherenkov detectors. H.E.S.S. has revealed the morphology of RX J1713.7-3946 SNR in the TeV domain, providing the first image of a TeV source (Aharonian et al. 2004), well correlated with the X-ray synchrotron morphology (Aharonian et al. 2006). However, the $\mathrm{TeV}$ gamma-ray emission can be produced either by accelerated electrons (Inverse Compton) or by accelerated protons (through pion decay following proton/proton collisions). The nature of which is a key question for particle acceleration as their respective energetic requirement is considerably different; a consistent modeling of the overall non-thermal spectrum is crucial to disentangle between the two possible origins.

\section{Nonthermal X-rays in Supernova remnants}

Nonthermal X-ray emission is observed in a number of supernova remnants over a relatively large range of parameters: from very young supernova remnants (like G1.9+0.3, 110 years old) to evolved remnants of above a thousand years (like RXJ1713-3946), from type Ia to core-collapse supernova remnants, from thermally-dominated remnants (like Tycho's SNR) to synchrotron-dominated remnants (like RXJ1713-3946).

\subsection{Synchrotron X-ray emission in young ejecta-dominated supernova remnants}

Chandra and XMM-Newton spatially-resolved spectroscopic studies of galactic historical supernova remnants have allowed to distinguish the emission arising from the shocked ejecta from that of the shocked interstellar medium. This was not affordable with previous X-ray instruments.

The first discovery has been on the nature of the emission associated with the shocked interstellar medium. While thermal emission was expected, the spectra of the blast wave were revealed to be featureless in Tycho's SNR (Hwang et al. 2002) and the morphology of the emission filamentary (see Fig. 2). In the framework of a thermal emission, the observed spectra implied a strongly out of ionization equilibrium plasma (to avoid line emission) with a high electronic temperature (of about $2 \mathrm{keV}$ ) all along the rim. For shock velocities of about $3000-4000 \mathrm{~km} / \mathrm{s}$, this was implying an electron-to-mean temperature ratio of $0.1-0.2$, which requires additional electron heating than solely through Coulomb interaction. On the other hand, a non-thermal synchrotron origin was consistent with the good agreement with the radio synchrotron morphology. The spectra, fitted by an 
exponential cut-off synchrotron model, gave values of the cut-off frequency of the order of 3 to $710^{16} \mathrm{~Hz}$. As shown in Figure 2, such featureless spectra were observed in the other historical young supernova remnants like Kepler's SNR (Cassam-Chenaï et al. 2004a) and Cas A (Vink \& Laming 2003).

On the basis of solely available spectra, it was not possible to distinguish between both possible interpretations (thermal vs nonthermal emission). The answer came from the interpretation of the morphology of the emission, notably the fact that the emission appears as narrow filaments, which was more naturally explained in the context of a nonthermal synchrotron emission. Such features of the blast wave X-ray emission were revealed by Chandra observations in a number of young supernova remnants: Cas A (Hughes et al. 2000, Gotthelf et al. 2001); Tycho (Hwang et al. 2002, Warren et al. 2005), Kepler (Bamba et al. 2005), SN 1006 (Bamba et al. 2003, Long et al. 2003). They were interpreted as synchrotron loss-limited filaments implying an amplified post-shock magnetic field (Vink \& Laming 2003, Ballet 2006, Völk, Berezhko \& Ksenofontov 2005, Parizot et al. 2006). Pohl, Yan \& Lazarian (2005) put forward an alternative interpretation, namely the damping of the magnetic field behind the shock. Both interpretations require significant magnetic field amplification at the shock, which is supported by theoretical studies (Lucek \& Bell 2000, Bell \& Lucek 2001, Bell 2004). These models predict slightly different synchrotron morphologies and spectral shapes in X-rays (Cassam-Chenaï et al. 2007, Rettig \& Pohl 2012).

The second discovery was to realize that the forward shock was lying much closer to the contact discontinuity than was expected from pure hydrodynamical models (Decourchelle 2005, Warren et al. 2005), implying that efficient particle acceleration was on going at the forward shock in young supernova remnants like Tycho and Kepler, as expected from

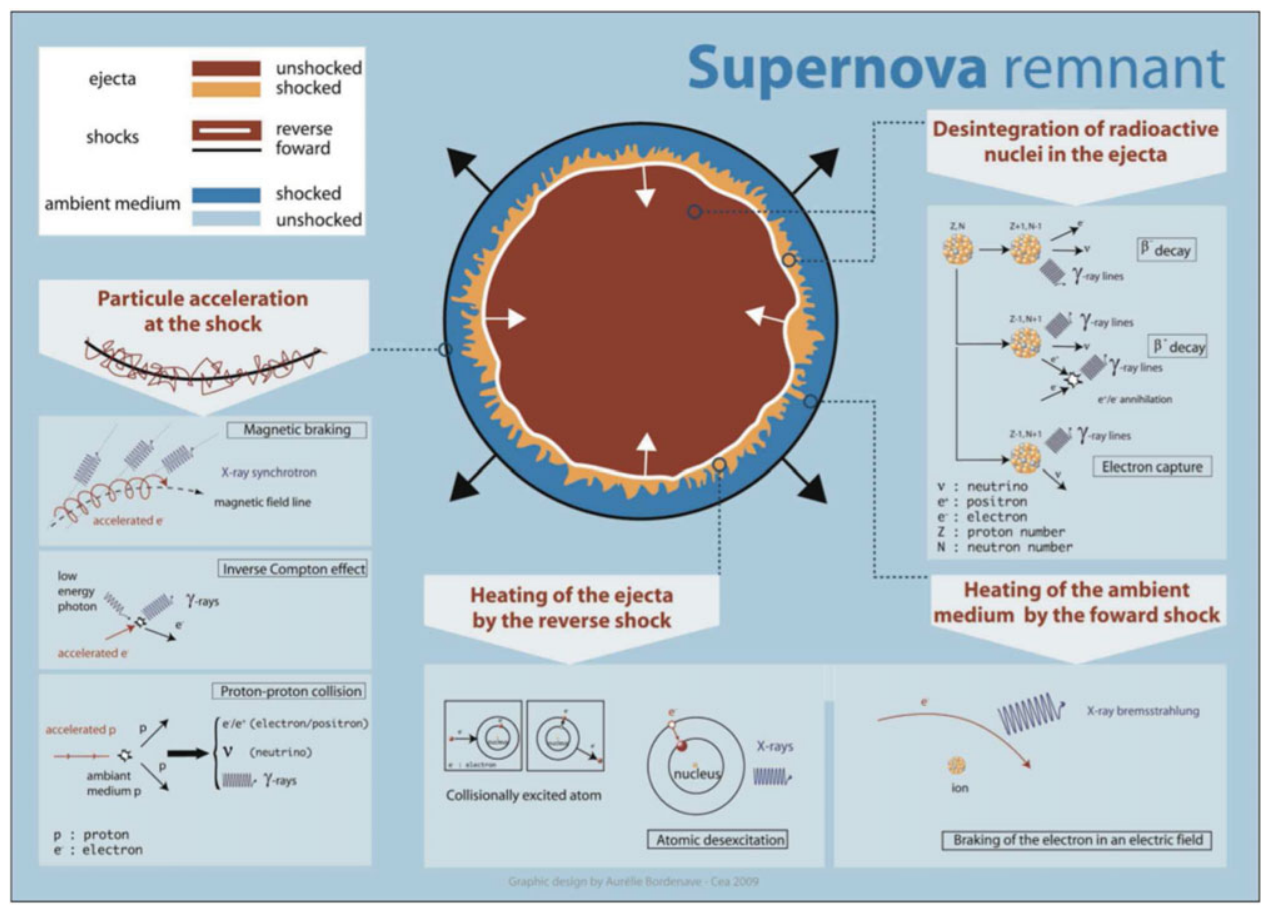

Figure 1. Sketch of the structure of a young supernova remnant, showing the main physical processes at work and the associated emission processes. 
hydrodynamical models including efficient particle acceleration at the shock (Chevalier 1983, Decourchelle, Ellison \& Ballet 2000).

\subsection{Synchrotron-dominated supernova remnants}

The first signature of $\mathrm{TeV}$ accelerated electrons in shell-type supernova remnants was obtained in SN 1006 with ASCA (Koyama et al. 1995). A second more extreme shell-type supernova remnant, RXJ 1713-3946, exhibiting only synchrotron emission was discovered in 1997 with $A S C A$ (Koyama et al. 1997). The source was first identified as a supernova remnant in 1996 by Pfeffermann \& Aschenbach (1996), based on ROSAT observations. The ASCA observations (Koyama et al. 1997, Slane et al. 1999) revealed a featureless X-ray spectrum well fitted by a power law model $(\gamma \simeq 2.4)$. No thermal emission was detected anywhere in the SNR implying a very low-density medium $\left(\leqslant 0.28 \mathrm{~cm}^{-3}\right.$, assuming a distance of $6 \mathrm{kpc}$ ). Assuming a magnetic field of $10 \mu \mathrm{G}$, the observed X-ray synchrotron emission arises from accelerated electrons at energies of $300 \mathrm{TeV}$. Further studies with $X M M-N e w t o n$ have enabled a complete characterization of the properties of the nonthermal emission. Combined with radio $\mathrm{CO}$ and $\mathrm{HI}$ data, absorption studies lead to reevaluate the distance of RXJ 1713-3946 to about $1 \mathrm{kpc}$ (see Cassam-Chenaï et al. 2004b) and were confirmed by dedicated CO observations (e.g., Fukui et al. 2003, Moriguchi et al. 2005). Combined X-ray and TeV gamma-ray spectral analysis were performed on this unique source to constrain the origin (leptonic versus hadronic) of the gamma-ray emission (Acero et al. 2009). A non-linear correlation between the X-ray and $\mathrm{TeV}$ gamma-ray flux were suggested by the data, which is easier to reproduce in the framework of a leptonic model. While SN 1006 and RXJ 1713-3946 are the best examples of this category of supernova remnants, X-ray synchrotron emission has been discovered in a number of other cases: RX J0852.0-4622 (Slane et al. 2001), RCW 86 (Bamba, Koyama \& Tomida 2000), G1.9+0.3 (Reynolds et al. 2008), G330.2+1.0 (Park et al. 2009), J1731-347 (Tian et al. 2010).

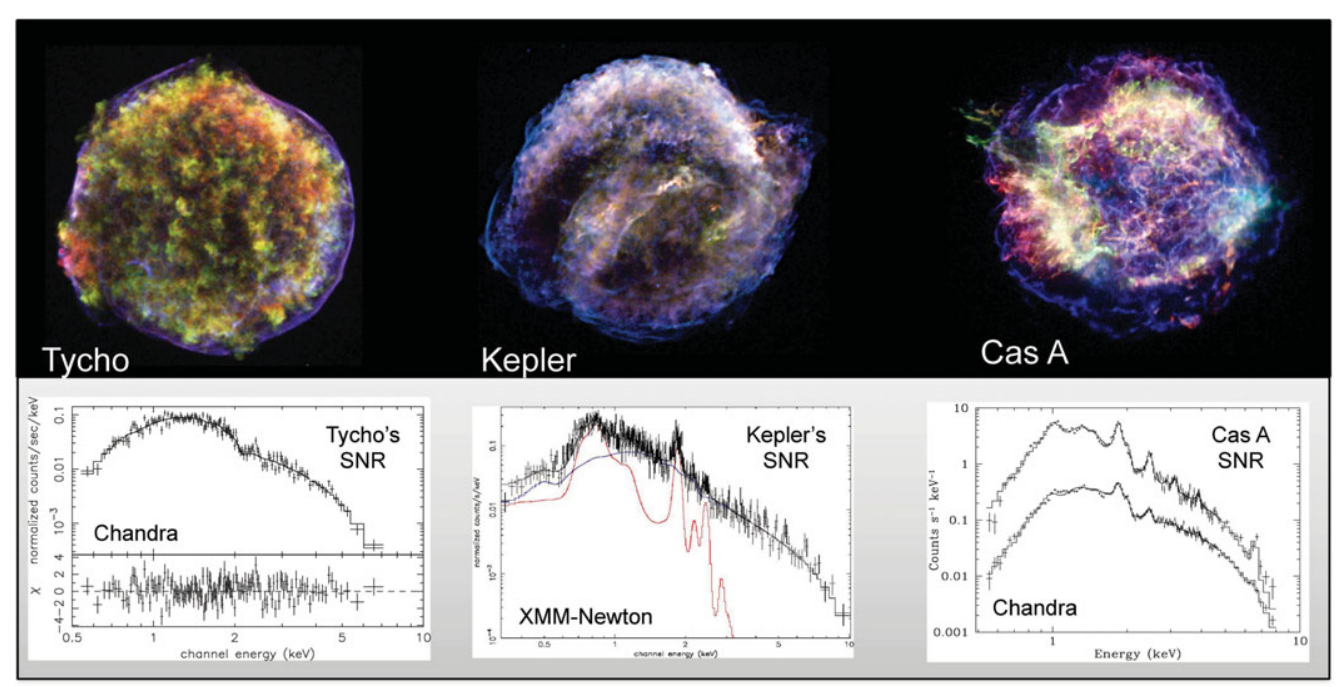

Figure 2. Composite 3-color Chandra images (top) of three young supernova remnants. The featureless spectra (bottom) of the narrow filamentary emission observed along the outer blast wave is shown to be synchrotron X-ray emission. Spectra from Hwang et al. (2002) for Tycho's SNR, Cassam-Chenaï et al. (2004a) for Kepler's SNR, and Vink \& Laming (2003) for Cas A SNR. 


\section{Constraints on particle acceleration from nonthermal X-ray emission in supernova remnants}

Since the discovery that cosmic rays are extra-terrestrial by Victor Hess in 1912, extensive observational and theoretical efforts have been made to understand their nature and origin. Their spectrum extends with relative regularity over 12 orders of magnitude in energy to about $10^{20} \mathrm{eV}$. Cosmic rays are mainly constituted of ions ( $87 \%$ protons, $12 \%$ helium, $1 \%$ heavier elements) and only $2 \%$ of them are electrons. While a number of characteristics of this population are now well known, the understanding of their origin remains a recalcitrant problem. It raises a number of distinct questions related to the origin of the energy, the acceleration mechanism at work, the maximum energy (Lagage \& Cesarsky 1983) and spectral form produced by this mechanism. The study of supernova remnants is crucial to answer these questions for cosmic rays below the knee at about $310^{15} \mathrm{eV}$. In the X-ray domain, the information comes primarily from synchrotron emission from accelerated electrons spiraling in the remnant magnetic field. While electrons are only a small fraction of cosmic rays, they can reveal a lot on the mechanism of diffusive shock acceleration, as they are accelerated almost like protons. Synchrotron X-rays arise from the highest-energy electrons $\left(10^{13} \mathrm{eV}\right.$ and more), and thus probe the cut-off energy in the electron distribution, the downstream magnetic field and indirectly the geometry of the ambient magnetic field. It allows to investigate a number of key questions: level of magnetic field amplification at the shock, maximum energy of the accelerated particles, efficiency of particle acceleration, as well as where particle acceleration occurs preferentially (quasi-perpendicular versus quasi-parallel acceleration), and how particle acceleration depends on ambient magnetic field orientation.

\subsection{Magnetic field}

The magnetic field is a crucial parameter for understanding particle acceleration, for deriving the maximum energy of accelerated particles from synchrotron cut-off frequency and for interpreting the origin of $\mathrm{TeV}$ gamma rays in term of leptonic versus hadronic emission.

It has been realized in the last ten years that the magnetic field could be significantly amplified at supernova remnant shocks. This is supported by theoretical approaches which predict magnetic field amplification at shocks, due to instabilities occurring for efficient particle acceleration (Lucek \& Bell 2000, Bell \& Lucek 2001, Bell 2004) or alternatively due to a preexisting turbulent medium (Giacalone \& Jokipii 2007). There are also a number of observational indications that the magnetic field is indeed amplified to the range $50-500 \mu \mathrm{G}$ :

- the sharpness of the synchrotron filaments at the forward shock (see Sect. 2.1).

- the fast variability of the brightness of some of these filaments (e.g., Patnaude \& Fesen 2009, Uchiyama et al. 2007, Uchiyama \& Aharonian 2008), although see Bykov et al. (2008) for an alternative interpretation.

- the broadband modeling of the nonthermal emission (e.g., Berezhko, Ksenofontov \& Völk 2012, Berezhko, Pühlhofer \& Völk 2009, Völk, Ksenofontov \& Berezhko 2008), see for an alternative Ellison et al. (2012).

The morphology of the synchrotron emission provides strong constraints on the geometry of the acceleration (Reynolds 1998). Reynolds (1996) proposed a model in which the asymmetry in SN 1006 is due solely to a large scale magnetic field aligned southeast to north-west. The Chandra data on the northeastern limb were interpreted in this framework where cosmic rays are accelerated identically everywhere (Long et al. 2003). Using $X M M-N e w t o n$ observations, the very low brightness of the emission in the interior 
above $2 \mathrm{keV}$ (mostly from synchrotron emission) was shown to be incompatible with an equatorial belt geometry and rather indicates that the bright non-thermal limbs are polar caps (Rothenflug et al. 2004). Berezhko, Ksenofontov \& Völk (2002, 2003) theoretically proposed such geometry of the upstream field. This implies that particles are accelerated where the magnetic field is parallel to the shock velocity in SN1006. 3D MHD simulations of the radio morphology are consistent with this interpretation (Bocchino et al. 2011).

\subsection{Maximum energy of accelerated particles}

From the measurements of the maximum energy of accelerated electrons and the downstream magnetic field, we can theoretically determine the maximum energy of accelerated protons. The measurement of the rolloff photon energy is related to the energy of the exponential cutoff in the electron spectrum, $E_{\max }=39\left(h \nu_{\mathrm{roll}} / B_{10}\right)^{1 / 2} \mathrm{TeV}$ (e.g., Reynolds et al. 2008), where $h \nu_{\text {roll }}$ is in $\mathrm{keV}$ and $B_{10}$ in units of $10 \mu \mathrm{G}$.

The highest measured rolloff frequency is observed in the youngest known galactic supernova remnant, G1.9+0.3. With a shock velocity of $\simeq 14000 \mathrm{~km} / \mathrm{s}$ and an age of about 110 years (Carlton et al. 2011), the featureless synchrotron emission reveals a rolloff frequency of $2.2 \mathrm{keV}$ (Reynolds et al. 2009). Assuming a moderate magnetic field of $10 \mu \mathrm{G}$, the maximum energy reaches about $70 \mathrm{TeV}$.

High latitude SNRs evolving in a uniform interstellar magnetic field, like SN 1006, offer the possibility to investigate the dependence of the process of particle acceleration with magnetic field orientation (see Sect.3.1). XMM-Newton observations suggested that the maximum energy of electrons was much larger at the bright limbs than elsewhere, so that the maximum energy reached by particles (not only their number) is higher at the bright limbs than in the southeast and northwest (Rothenflug et al. 2004, Miceli et al. 2009). The observed averaged azimuthal variation of the rolloff frequency along the shock is very strong (factor 10) and cannot be explained by variations of the magnetic compression alone. A more detailed study is however required to get a proper estimate of the rolloff energy of the synchrotron spectrum at the spatial scale of the filamentary structures all along the shock (using radio data to anchor the spectrum at low energy). This is one of the objective of a large XMM-Newton program of observation of SN1006. First results on the shape of the cutoff in the synchrotron spectrum indicates that a loss-limited model provides the best match to the data (Miceli et al. 2013).

\subsection{Efficiency of particle acceleration}

Depending on the efficiency of particle acceleration at the shock, the synchrotron spectrum is expected to differ in shape. Test-particle acceleration models predict a power-law synchrotron emission, while nonlinear diffusive shock acceleration predict a curvature of the particle spectra (e.g., Ellison \& Reynolds 1991). A few attempts have been made to search for curvature in the synchrotron spectra of supernova remnants. Some indications of curvature were found combining radio and X-ray data in SN 1006 (Allen et al. 2008) and RCW 86 (Vink et al. 2006), using infrared data in Cas A (Jones et al. 2003) and from radio data for Tycho and Kepler (Reynolds \& Ellison 1992).

\section{Conclusion}

Nonthermal X-ray emission from supernova remnants provides crucial diagnostics to constrain the magnetic field intensity and geometry, and determine the properties of particle acceleration at their shock. Such studies require spatially resolved X-ray spectroscopy. Large observing programs, with XMM-Newton or Chandra satellites, are required to obtain sufficient statistics at the relevant spatial scale to characterize the properties of the 
narrow shock structure and its possible variability. There is a clear need for the extension of the operations of XMM-Newton and Chandra satellites, as well as a need for the preparation of the next X-ray observatory Athena+.

A consistent approach, including both thermal and nonthermal X-rays as well as broadband nonthermal observations from radio to $\mathrm{TeV}$ gamma rays, is required to fully constrain the parameters of the acceleration of particles at shocks in supernova remnants. These are key elements to understand the origin of Galactic cosmic rays.

\section{References}

Acero, F., Ballet, J., Decourchelle, A., Lemoine-Goumard, M., Ortega, M. et al. 2009, A\&A, 505,157

Aharonian, F., Akhperjanian, A. G., Aye, K.-M., Bazer-Bachi, A. R., Beilicke, M. et al. 2004, Nature, 432,75

Aharonian, F. A., Akhperjanian, A. G., Bazer-Bachi, A. R., Beilicke, M., Benbow, W., Berge, D., Bernlhr, K., Boisson, C., Bolz, O., Borrel, V. et al. 2006, A\&\&A, 449, 223

Allen, G. E., Houck, J. C., \& Sturner, S. J. 2008, ApJ, 683, 773

Ballet, J. 2006, AdSR, 37, 1902

Bamba, A., Koyama, K., \& Tomida, H. 2000, PASJ, 52, 1157

Bamba, A., Yamazaki, R., Ueno, M., \& Koyama, K. 2003, ApJ, 589, 827

Bamba, A., Yamazaki, R., Yoshida, T., Terasawa, T., \& Koyama, K. 2005, ApJ, 621, 793

Bell, A. R. 2004, MNRAS, 353, 550

Bell, A. R. \& Lucek, S. G. 2001, MNRAS, 321, 433

Berezhko, E. G., Ksenofontov, L. T., \& Völk, H. J. 2002, A\&A, 395, 943

Berezhko, E. G., Ksenofontov, L. T., \& Völk, H. J. 2003, $A \& A$, 412, L11

Berezhko, E. G., Ksenofontov, L. T., \& Völk, H. J. 2012, ApJ, 759, 12

Berezhko, E. G., Pühlhofer, G., \& Völk, H. J. 2009, A\&A, 505, 641

Blandford, R. \& Eichler, D. 1987, Phys.Rep., 154, 1

Bocchino, F., Orlando, S., Miceli, M., \& Petruk, O. 2011, A\&A, 531, 129

Bykov, A. M., Uvarov, Y. A., \& Ellison, D. C. 2008, ApJ, 689, L133

Carlton, A. K., Borkowski, K., Reynolds, S. P., Hwang, U., Petre, R., Green, D. A., Krishnamurthy, K., \& Willett, R. 2011, ApJ, 737, L22

Cassam-Chenai, G., Decourchelle, A., Ballet, J., Hwang, U., Hughes, J. P., \& Petre, R. 2004, $A \& A, 414,545$

Cassam-Chenai, G., Decourchelle, A., Ballet, J., Sauvageot, J.-L., Dubner, G., \& Giacani, E., 2004, $A \mathscr{E} A, 427,199$

Cassam-Chenaï, G., Hughes, J. P., Ballet, J., \& Decourchelle, A. 2007, ApJ, 665, 315

Chevalier, R. A. 1983, ApJ, 272, 765

Decourchelle, A. 2005, in: L.O. Sjouwerman \& K. K Dyer (eds.), X-Ray and Radio Connections(Published electronically by NRAO, http://www.aoc.nrao.edu/events/xraydio), E4.02, 10 pages

Decourchelle, A., Ellison, D. C., \& Ballet, J. 2000, ApJ, 543, L57

Ellison, D. C. \& Reynolds, S. P. 1991, ApJ 382, 242

Ellison, D. C., Slane, P., Patnaude, D. J., \& Bykov, A. M. 2012, ApJ, 744, 39

Fukui, Y., Moriguchi, Y., Tamura, K., Yamamoto, H., Tawara, Y. et al. 2003, PASJ, 55, L61

Giacalone, J. \& Jokipii, J. R. 2007, ApJ, 663, L41

Gotthelf, E. V., Koralesky, B., Rudnick, L., Jones, T. W., Hwang, U., \& Petre, R. 2001, ApJ, 552 , L39

Hanbury Brown, R. 1954, The Observatory, 74, 185

Hughes, J. P., Rakowski, C. E., Burrows, D. N., \& Slane, P. O. 2000, ApJ, 528, L109

Hwang, U., Decourchelle, A., \& Holt, S. S., Petre R. 2002, ApJ, 581, 1101

Jones, T. J., Rudnick, L., DeLaney, T., \& Bowden, J. 2003, ApJ, 587, 227

Koyama, K., Kinugasa, K., Matsuzaki, K., Nishiuchi, M., Sugizaki, M. et al. 1997, PASJ, 49, L7 
Koyama, K., Petre, R., Gotthelf, E. V., Hwang, U., Matsuura, M., Ozaki, M., \& Holt, S. S. 1995, Nature, 378, 255

Lagage, P. O. \& Cesarsky, C. J. 1983, A\&A 125, 249

Lemoine-Goumard, M. 2013, this proceedings

Long, K. S., Reynolds, S. P., Raymond, J. C., Winkler, P. F., Dyer, K. K., \& Petre, R. 2003, ApJ 586, 1162

Lucek, S. G. \& Bell, A. R. 2000, MNRAS, 314, 65

Miceli, M.; Bocchino, F., Decourchelle, A., Vink, J., Broersen, S., \& Orlando, S. 2013, A\&A in press, astro-ph ariv:1306.6048

Miceli, M., Bocchino, F., Iakubovskyi, D., Orlando, S., Telezhinsky, I., Kirsch, M. G. F., Petruk, O., Dubner, G., \& Castelletti, G. 2009, A\&SA 501, 239

Moriguchi, Y., Tamura, K., Tawara, Y., Sasago, H., Yamaoka, K. et al. 2005, ApJ, 631, 947

Parizot, E., Marcowith, A., Ballet, J., \& Gallant, Y. A. 2006, A\& A, 453, 387

Park, S., Kargaltsev, O., Pavlov, G. G., Mori, K., Slane, P. O., Hughes, J. P., Burrows, D. N., \& Garmire, G. P. 2009, ApJ, 695, 431

Patnaude, D. J. \& Fesen, R. A. 2009, ApJ, 697, 535

Pfeffermann, E. \& Aschenbach, B. 1996, in: Zimmermann, Trümper and Yorke (eds.), Roentgenstrahlung from the Universe, 267

Pohl, M., Yan, H., \& Lazarian, A. 2005, ApJ, 626, L101

Rettig, R. \& Pohl, M. 2012, A\&A, 545, 47

Reynolds, S. P. 1996, ApJ, 459, L13

Reynolds, S. P. 1998, ApJ, 493, 375

Reynolds, S. P., Borkowski, K. J., Green, D. A., Hwang, U., Harrus, I., \& Petre, R. 2008, ApJ, 680, L41

Reynolds, S. P., Borkowski, K. J., Green, D. A., Hwang, U., Harrus, I., \& Petre, R. 2009, ApJ, 695, L149

Reynolds, S. P. \& Ellison, D. C. 1992, ApJ 399, L75

Rothenflug, R., Ballet, J., Dubner, G., Giacani, E., Decourchelle, A. et al. 2004, A\&A, 425, 121

Slane, P. 2013, this proceedings

Slane, P., Gaensler, B. M., Dame, T. M., Hughes, J. P., Plucinsky, P. P., \& Green, A. 1999, ApJ, 525,357

Slane, P., Hughes, J. P., Edgar, R. J., Plucinsky, P. P., Miyata, E., Tsunemi, H., \& Aschenbach, B. $2001, A p J, 548,814$

Tian, W. W., Li, Z., Leahy, D. A., Yang, J., Yang, X. J. et al. 2010, ApJ, 712, 790

Uchiyama, Y., Aharonian, F. A., Tanaka, T., Takahashi, T., \& Maeda, Y. 2007, Nature, 449, 576

Uchiyama, Y. \& Aharonian, F. A. 2008, ApJ, 677, L105

Vink, J. \& Laming, J. M. 2003, ApJ, 584, 758

Vink, J., Bleeker, J., van der Heyden, K., Bykov, A., Bamba, A. et al. 2006, ApJ, 648, L33

Völk, H. J., Berezhko, E. G., \& Ksenofontov, L. T. 2005, A\&A, 433, 229

Völk, H. J., Ksenofontov, L. T., \& Berezhko, E. G., 2008, A\&SA490, 515

Warren, J. S., Hughes, J. P., Badenes, C., Ghavamian, P., McKee, C. F., Moffett, D., Plucinsky, P. P., Rakowski, C., Reynoso, E., \& Slane, P. 2005, ApJ 634, 376

\section{Discussion}

MitRA: What is the expression for the maximum energy of cosmic rays? How do you get the magnetic field?

DeCOURCHELle: The expression of the maximum energy of electrons is given in sect. 3.2. It is derived from synchrotron physics and assumes an exponential cutoff in the electron spectrum. The maximum energy of cosmic rays depends on the limiting mechanism. If radiative losses cutoff the electron spectrum, accelerated protons can have much higher maximum energy. The magnetic field is derived from the width of the narrow X-ray synchrotron loss-limited filaments along the shock (Vink \& Laming 2003). 